(e-migrinter

\author{
e-Migrinter
}

$10 \mid 2013$

Genre et imbrication des rapports de domination dans les médias des minorités ethniques

\title{
Médias panafricains francophones et reproduction des rapports de pouvoir - Années 1980/1990
}

\section{Carmen Diop}

\section{OpenEdition}

\section{Journals}

Édition électronique

URL : https://journals.openedition.org/e-migrinter/481

DOI : 10.4000/e-migrinter.481

ISSN : 1961-9685

Éditeur

UMR 7301 - Migrinter

Édition imprimée

Date de publication : 11 avril 2013

Pagination : 61-78

ISSN : 1961-9685

\section{Référence électronique}

Carmen Diop, « Médias panafricains francophones et reproduction des rapports de pouvoir - Années 1980/1990 », e-Migrinter [En ligne], 10 | 2013, mis en ligne le , consulté le 20 mai 2021. URL : http:// journals.openedition.org/e-migrinter/481; DOI : https://doi.org/10.4000/e-migrinter.481 
 \\ Point de vue situé : Médias panafricains francophones et reproduction des rapports de pouvoir - Années 1980/1990*}

\section{Carmen Diop}

J

ournaliste dans les médias de la diaspora africaine francophone pendant quinze ans, j'ai observé leurs organisations et leurs pratiques. Cet article interroge les rapports de pouvoir dans les rédactions des médias minoritaires ${ }^{1}$ et tente de comprendre leurs spécificités en soulignant la question du genre ${ }^{2}$, de la race $^{3}$ et de l'ethnicité ${ }^{4}$. Il s'appuie sur la perspective du point de vue situé, la sociologie des professions et du journalisme, ainsi que sur une enquête de terrain.

\footnotetext{
*Je remercie les coordinatrices de cette publication, en particulier Claire Cossée, pour leur soutien et leurs remarques.

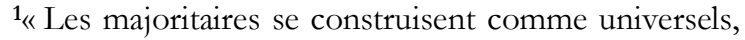
ils incarnent la norme et imposent leurs normes (Guillaumin, 1972, pp.115-128). Ils confinent ainsi les minoritaires dans leur différence, leur imposant l'universalisme, ou, plus précisément, leur propre spécificité » (Sosoe, 2002, p.66).

${ }^{2}$ Différences sexuées socialement construites.

${ }^{3}$ Caractéristiques visibles sélectionnées pour naturaliser une hiérarchie sociale (Guillaumin, 1972). Ce terme est utilisé sans guillemets.

${ }_{4}$ L'ethnicité est une croyance en des ancêtres communs, réels ou putatifs. [...] Les frontières, qui incluent tant les dimensions institutionnelles que les représentations, ne sont jamais tangibles. [...] la spécificité des relations ethniques et leur autonomie relative face aux classes sociales; les relations ethniques appréhendées comme des relations non entre des groupes économiques, mais entre des groupes de statut unis par des liens économiques, politiques et idéologiques; l'importance de la culture et de l'histoire, et l'humanisation des êtres humains comme processus d'ethnicisation [doivent être questionnées]» (Juteau, 1999, p.15).
}

Je montre dans cet article comment les rapports de pouvoir Nord/Sud se doublent de rapports de sexe, de classe ${ }^{5}$, voire de caste $^{6}$. Je m'attarde sur l'« entre soi» qui structure l'organisation des médias et sur les rapports de pouvoir dans les rédactions. Mon hypothèse est que, sous couvert de s'adresser à un public africain avec des patrons de presse et des rédacteurs africains, ces médias reproduisent le modèle des rapports sociaux de sexe de la presse dominante, ainsi que le déséquilibre Nord/Sud dans leurs modes de production et ce dans un contexte néocolonial ${ }^{7}$ (Sartre, 1956 ; Nkrumah, 1965 ; Ardant, 1965 ; Mazrui, Mutunga, 2003).

\section{Cadres théoriques de cette restitution réflexive d'un vécu professionnel}

Je m'appuie sur l'épistémologie du point de vue qui postule que la connaissance est située, partielle et partiale (Juteau, 1981) et que le savoir dominant androcentré occulte sa propre position (Mathieu, 1971; 1991; Haraway, 1988). Les théories anticoloniale, postcoloniale, le féminisme hégémonique, la transversalité des catégories sociales, les politiques des identités et du corps se réfèrent au savoir situé (Preciado, 2005). La conceptualisation de l'oppression doit venir de l'opprimé (Delphy, 1998, 2001) dont les analyses doivent être pensées en

\footnotetext{
${ }^{5}$ Groupe social inscrit dans une relation de domination avec d'autres groupes.

${ }^{6}$ Groupe social endogame et héréditaire. Les éléments biographiques en notes de bas de page soulignent la « consanguinité » de ce milieu.

${ }^{7}$ Méthodes d'influence insidieuses et complexes des anciennes puissances coloniales sur des États théoriquement indépendants.
} 
termes de "privilège épistémique » (Hartsock, 1998). Les inconvénients en terme d'oppression deviennent des avantages en terme de science (Harding, 1990 ; 2003) et l'acteur engagé peut s'appuyer sur sa colère pour produire des "effets théoriques" dans le champ scientifique (Guillaumin, 1981; 1992). La marginalité est un «lieu de résistance» à «l'académisme blanc» (hooks, 2000) et les positionnalités doivent être négociées sans être rassemblées sous la seule catégorie du genre (Gutiérrez Rodríguez, 2006). Le point de vue situé renvoie aux valeurs personnelles, à l'objectivité, à la rationalité et à l'autorité épistémique. Mon positionnement me permet d'interroger les relations sociales de domination dans les médias ainsi que les rapports sociaux ${ }^{8}$ qui en découlent.

Pour montrer comment les médias panafricains francophones sont traversés par les rapports de domination néocoloniale, je m'appuie également sur la pensée postcoloniale qui analyse les déséquilibres issus de l'impérialisme (Fanon, 1952; Memmi, 1957 ; Saï, 1980 ; Spivak, 2006 ; Bhabha, 2007) et sur les études médiatiques postcoloniales (Parameswaran, 2001, 2002a, 2002b, 2008 ; Wasserman, Rao, 2008 ; Hall, 2012; Brisset-Foucault, 2009). Il faut également noter que les journalistes sont soumis aux contraintes de production de l'information. De plus, des groupes hétérogènes, qui partagent normes et routine professionnelles (Bourdieu, 1971), cohabitent dans les médias. L'idéologie dominante conditionne la hiérarchie de l'information et l'interprétation des faits (Bourdieu, 2000). J'interroge les conditions qui font obstacle à l'innovation dans les médias minoritaires (De Lagasnerie, 2011) et à la transgression des normes éthiques.

${ }^{8}$ Kergoat (2009) distingue rapport social, d'ordre théorique, et relation sociale, d'ordre empirique. Le rapport social précède les interactions, en découle, et évolue avec les pratiques, alors que les relations sociales n'influencent pas les rapports sociaux.
Le concept d'intersectionnalité, également mobilisé, (Delphy, 2001; Guillaumin, 1972 ; Juteau, 1999 ; Dorlin, 2008 ; Kergoat, 2009) a permis de sortir les femmes noires de l'invisibilité sociale (Bilge, 2009a) : il m'aide à comprendre l'intrication et les configurations des rapports de domination auxquels elles sont soumises dans ce milieu professionnel, comment genre et race se conjuguent dans la division du travail, et comment "les rapports de genre exacerbent les rapports de classe» (Kergoat, 2009, p.125).

\section{Organisation économique et « entre soi » (post) colonial}

Depuis un demi-siècle, la presse panafricaine francophone gravite autour de la figure du newsmagazine Jeune Afrique (JA). « [...] un journal, [...] même s'il est pauvre, doté de peu de ressources et disposant de moyens limités, il arrive à tenir et à s'en sortir. $[\ldots] \quad[s$ '] il $[\ldots]$ équilibre $[\ldots]$ les revenus et les charges. [...] les études que j’ai faites [HEC, NDR] me permettent de savoir [...] comment bien gérer une entreprise de presse. [...]. Ceux qui ont essayé de créer des hebdomadaires ou d'autres publications n'étaient pas tous des gestionnaires. [...]. Jean-Baptiste Placca, pour L'Autre Afrique, il aurait dû s'attacher les services d'un gestionnaire [...]. Idem pour Demain l'Afrique: Paul Bernetel s'est cassé les dents sur la gestion. [...] [L'un des] secret[s] de [la] longévité [de $J A$ ], c'est que j'ai eu la chance de pouvoir cumuler les deux fonctions principales d'un journal: la gestion et la rédaction en chef. J'ai quitté la politique, j'ai quitté les affaires, j’ai tout quitté pour ne faire que cela depuis cinquante ans. [...] Le cumul des deux fonctions est un grand atout, mais c'est aussi un inconvénient sérieux, parce qu'un rédacteur en chef est porté sur la dépense, alors qu'un directeur de gestion est fortement enclin à la freiner. Ayant été davantage rédacteur en chef, j’ai souvent commis l'erreur de trop dépenser » (Boisbouvier, 2010, [1/5]). 
Outre les problèmes strictement économiques, les médias panafricains francophones sont traversés par des enjeux de pouvoir multiformes. Les $N M P P^{9}$, qui assurent la diffusion internationale des journaux, imposent leurs conditions financières. Le contexte global, comme la dévaluation du Franc CFA joue un rôle important. " $J A$ a failli mourir entre 1994 et la fin du siècle. La moitié de nos lecteurs n'avaient plus les moyens d'acheter le journal et presque tous nos annonceurs nous avaient abandonnés. $J A$ perdait 1,5 million de francs français par mois. [...] Nous ne pouvions même plus payer le loyer et les salaires. Les ventes de $J A$ sont passées de 150000 exemplaires à moins de 40000 . [...] les effectifs du journal ont chuté de 120 à 30 personnes. [...]. Nos salariés nous ont aidés: [en] renon[çant] à une partie de leur salaire. Nos actionnaires - $[\ldots] 550$ [dans] cinquante-cinq pays - nous ont aussi apporté leur soutien [en mettant] un peu d'argent sans toucher [...] de dividendes. [...] Nous avons traversé sept années de cauchemar, mais nous nous sommes progressivement redressés. » (Boisbouvier, 2010, [2/5]).

Les groupes Jeune Afrique, Gideppe (Jeune Afrique Économie (JAE) et Divas) et Elite International (Afrique Élite et Élite Madame) ont tenté des concentrations qui ont contribué à réduire le pluralisme et l'indépendance de l'information. Certains médias sont financés par des gouvernements, comme la radio Africa $N^{\circ} 1$, créée en 1981 par le président gabonais Omar Bongo et la SOFIR $A D^{10}$. D'autres $(J A E, J A$, etc.) proposent régulièrement des "dossiers pays» dont le contenu est contrôlé par des responsables politiques de la «Françafrique ${ }^{11}$ ». «Nos censeurs $[\ldots]$ s'accommodent mal de

${ }^{9}$ Les Nouvelles Messageries de la Presse Parisienne (maintenant Presstalis) contrôlent 80\% du marché depuis les années 1960.

${ }^{10}$ Gère les participations françaises dans l'audiovisuel, détenait 40\% d'Africa $N^{\circ} 1$ jusqu'en 2002.

${ }^{11}$ Réseaux français dans les anciennes colonies africaines. l'audience du journal dans leur pays qu'ils considèrent comme une intrusion dans leur pré carré. » (Boisbouvier, 2010, [2/5]). Ainsi, des gouvernements peuvent faire traîner les accréditations de correspondants (Africa $N^{\circ} 1$ ) ou les accorder séance tenante (Radio France Internationale - RFI), ou encore faire retirer certains numéros des kiosques. «Ce qui nous a fait le plus de mal [...], ce sont les saisies et les interdictions. En Guinée, nous avons été interdits [...] à partir de 1963 [...] jusqu'[en] 1983. En 1976, c'était au tour de l'Algérie, et ce pendant seize ans. [...] Les dernières interdictions remontent à la Côte d'Ivoire de Houphouët-Boigny et à la Tunisie d'Habib Bourguiba, dans les années 1980. Ce fut la période la plus périlleuse, car ces deux pays étaient deux marchés importants, à la fois pour la vente et la publicité. Ils ne l'ont pas su, mais ils ont failli nous tuer $[\ldots]$. En Algérie, $J A$ vendait chaque semaine 30000 exemplaires et avait 8000 abonnés. Cela représentait plus du tiers de notre diffusion. On avait un bureau à Alger et de nombreux annonceurs. [...] Le président Boumédiène a cru qu'il allait avoir la peau de Jeune Afrique [...]. » (Boisbouvier, 2010, [1/5]).

Un bref panorama du paysage médiatique panafricain francophone permet d'en situer les acteurs et de comprendre les rouages de l'influence persistante de l'ancienne puissance coloniale. Africa International a été créé à Dakar en 1958, par J. Decupper, publicitaire blanc ${ }^{12}$ installé au

${ }^{12}$ Le qualificatif « blanc » est utilisé par opposition à « noir » et autres caractères « ethnicisés ». Cette opposition confond ethnicité, origine géographique et phénotype, mais rend compte de la perception des acteurs sociaux. Tout comme les termes «race» et « noir », il est utilisé sans guillemets pour marquer un " univers sémantique [...] opérateur politique et symbolique d'assignations, et de désignations normatives facteurs d'altérisation et d'oppression» (Guénif-Souilamas, 2012). Le terme «européen » n'est pas utilisé ici, car il apparaît comme l'euphémisation d'une catégorie ethno-raciale et des nationalités des anciens colonisateurs qui sont pointés ici (France et, dans une moindre mesure, Belgique). Si 
Sénégal avant l'indépendance avec des journalistes africains. Maintenant adressé aux milieux politiques et d'affaires, il a été rapatrié à Paris en 1991 par la camerounaise M-R. Biloa ${ }^{13}$. En 1988, B-P. Talla, autodidacte camerounais ${ }^{14}$, rachetait $J A E^{15}$ à B. Ben Yahmed, fondateur franco-tunisien du Groupe $J A$ en 1967, qui estime aujourd'hui s'être trompé en lui faisant confiance. "Cela lui est monté à la tête et, on le sait maintenant, il a voulu s'en servir pour tuer Jeune Afrique. Depuis plus de vingt ans, il nous intente des procès et a dépensé une fortune en honoraires d'avocat. Il n'a pas réussi, mais j'ai tout de même commis une grave erreur en le choisissant lui et surtout en louant le titre. Ce que je voulais,

les Antillais utilisent le terme "métropolitain» pour désigner les personnes blanches originaires de l'Hexagone ; sur le continent, il s'agit bien de pointer la couleur de peau des anciens colons. Les deux termes s'adressent à des situations géographiques, culturelles, historiques et politiques différentes. Parler de «métropolitains » dans le contexte africain n'est donc pas pertinent, car non seulement le terme n'y est pas (plus, depuis les indépendances) utilisé, mais on ne peut pas y transposer la diversité des situations sociales des Blancs qui existe dans les Dom-Tom et qui justifie, entre autre, l'emploi de ce qualificatif pour les ressortissants blancs de France métropolitaine. Dans les deux cas, «métropolitain » et «blanc » sont investis de la charge des rapports de domination coloniaux et post-coloniaux. Le terme de "blanc" repris ici est souvent utilisé en Afrique dans le langage courant pour désigner les Européens et/ou les Occidentaux en les renvoyant à leur ancien statut de "colons". La citation de Béchir Ben Yahmed (p.65) rend bien compte de la manière dont cette opposition est articulée : il s'agit en effet d'un antagonisme à comprendre dans un cadre où le «centre » continue encore largement à façonner la sensibilité des « périphéries » à travers les productions culturelles.

${ }^{13}$ Elle figure dans l'Encyclopédie Ekang comme membre de l'ethnie Beti du Cameroun (celle du président Biya) et du peuple Ekang d'Afrique centrale. Elle dirige le magazine people Ici les gens $d u$ Cameroun, des think tanks, Club Millenium et Millenium Lady's Club. Elle est membre de l'Initiative pour le Développement des Médias en Afrique.

${ }^{14}$ Emprisonné par P. Biya dans les années 1980.

${ }^{15}$ Maintenant domicilié au Cameroun. c'était aider un Subsaharien à devenir un bon éditeur ${ }^{16} \gg$ (Boisbouvier, 2010, [2/5]). L'épouse métropolitaine de cet ancien secrétaire d'État à l'Information du président Bourguiba, ainsi que leurs enfants, occupent des postes clés dans les médias panafricains.

M. de Breteuil à créé Amina à Dakar en 1972. Il a succédé à son père, le comte C. de Breteuil, créateur de la $S A P E F^{17}$. En 1974, Amina encourageait à voter pour Senghor ${ }^{18}$. Les manœuvres des jeunes actionnaires des bourgeoisies locales d'Élite International pour se débarrasser de certains journalistes étaient tolérées par J-P. Pont ${ }^{19}$, créateur blanc du groupe. Le magazine féminin a alors été animé par E. Pontié, pigiste blanche, puis par l'antillaise M-J. Serbin. Fille de fonctionnaires martiniquais au Sénégal et ancienne chroniqueuse de la jet-set pour Africa International, elle a créé Divas (Gideppe), puis, avec N. Bat-Mensah, fille d'un diplomate béninois aux NationsUnies, Brune ${ }^{20}$.

Certains médias français tiennent une place importante dans ce paysage. La banque d'images de l'AITV (Agence Internationale d'Images de Télévision) conserve une grande partie de la mémoire télévisuelle de l'Afrique francophone ${ }^{21} . R F I^{22}$, qui dispute ses auditeurs à Africa $N^{\circ} 1$, diffusait Multiplex, un recueil de notes professionnelles en Afrique

\footnotetext{
${ }^{16}$ Signe des rivalités entre médias minoritaires et médias mainstream (RFI/Africa $N^{\circ} 1$ ) et avec les actionnaires mainstream (Sofirad/Africa $N^{\circ} 1$ ).

${ }^{17}$ Administrateur colonial créateur du premier quotidien d'Afrique noire en 1933, devenu l'organe officiel sénégalais en 1970. Amina est aujourd'hui gérée par Nathalie et Nicolas de Breteuil, tandis que leur père a lancé, en 2011, La revue littéraire du monde noir.

18Président du Sénégal de 1960 à 1980.

${ }^{19}$ Auteur de Voyage à l'Élysée (2007), ainsi que d'un répertoire de la coopération française et directeur de Vivre à l'Étranger destiné aux coopérants français.

${ }^{20}$ Brune organisait en 2012 une rencontre entre des entrepreneuses noires du web et ses lectrices... au ministère français de l'Outre-mer.

${ }^{21}$ Fondée en 1985 par RFO (Société de Radiodiffusion et de Télévision Française pour l'Outre-mer).

${ }^{22}$ L'ex Poste Colonial (1931), devenu Office français de Coopération Radiophonique (OCORA), puis RFI en 1975.
} 
francophone et dans l'océan Indien ${ }^{23}$. La légitimité du rédacteur en chef de Panos Infos $^{24}$, autre bulletin professionnel, semble avoir surtout reposé sur son capital familial $^{25}$. Le groupe Le Moniteur publiait la Lettre Afrique Expansion destinée à des investisseurs français.

Il apparaît que tous ces médias, minoritaires ou majoritaires spécialisés, étaient détenus par une élite fondée sur un capital économique et social postcolonial. Cet article a pour ambition de montrer comment la division du travail et la production de l'information $y$ reproduisaient l'hégémonie coloniale.

\section{Rapports de pouvoir dans les salles de rédaction}

Pour les tenants du néocolonialisme, la domination économique et culturelle sur les anciennes colonies qui se poursuit depuis les indépendances est maintenue par des moyens détournés. Les médias permettent d'étudier comment la production de l'information rend compte des liens persistants entre le «centre» («ancienne» métropole) et ses «marges» (pays «anciennement» colonisés). Ainsi, ceux qui sélectionnent l'information et imposent le style rédactionnel sont souvent des ressortissants de l'ex-puissance coloniale. L'éditorial de B-P. Talla dans $J A E$ était écrit par son ex-rédacteur en chef, devenu son conseiller $^{26}$. Et si c'est au directeur de l'information gabonais ${ }^{27}$ que j'ai envoyé ma

\footnotetext{
${ }^{23}$ Dirigé par J-P. Chailleux (directeur des programmes régionaux de France 3) et animé par B. Schoeffer (conseiller de radios africaines depuis 1958, puis conseiller du président de RFI) et le collectionneur d'art G. Robert.

${ }^{24}$ L'Institut Panos appuie le pluralisme et l'expression des populations marginalisées.

${ }^{25} \mathrm{O}$. Colombani, issu d'une famille d'administrateurs coloniaux.

${ }^{26}$ J-P. Béjot, rédacteur en chef d'un journal électronique publié par un homme d'affaires sénégalais.

27J. Mbourou, ancien directeur de la Radiodiffusion Télévision Gabonaise.
}

candidature, ma position de correspondante d'Africa $N^{\circ} 1$ à Dakar a été confirmée par un conseiller métropolitain ${ }^{28}$. Deux modèles se partagent ce paysage. Le groupe $J A$, qu'aucun concurrent n'a sérieusement inquiété pendant cinquante ans, fait figure d'exemple. «Ceux qui ont tenté de créer des journaux concurrents sont tous issus de Jeune Afrique. Ils y ont appris le métier, sont ses enfants » (Boisbouvier, 2010, [1/5]). À Jeune Afrique Magazine (JAM), virtuellement dirigé par Mme Ben Yahmed, les journalistes africains étaient encadrés par un staff éditorial $^{29}$ et technique métropolitain. Une situation que ne partageaient pas les plumes reconnues : «Les Subsahariens qui arrivent à Jeune Afrique se sentent chez eux. [...] Tout au long de son histoire et jusqu'à ce jour, Jeune Afrique a compté dans ses rangs des Africains -, noirs et blancs [...]. Cela marche assez bien parce que Jeune Afrique n'est pas un journal de Blancs qui emploient des Noirs. [...] Et quand un Siradiou Diallo, un Justin Vieyra ou un Sennen Andriamirado quittent les leurs pour venir travailler avec nous, $J A$ devient leur journal et leur famille » (Boisbouvier, 2010, [1/5]).

Cinq ans après avoir rejoint des rédactions moins formatées, je réécrivais les articles de $J A E$ pour en homogénéiser le style, sur le modèle éditorial de $J A \ldots$ Sans conférence de rédaction, les rédacteurs n'ont pas de prise sur les décisions. À Amina, M. de Breteuil avait un droit de regard absolu, sans intervention de la rédactrice en chef $^{30}$. A. Baruch réalisait entièrement Black, et ne déléguait que les articles à des pigistes qui se rencontraient ainsi par hasard. Panos Infos et la Lettre Afrique Expansion connaissaient aussi une forte centralisation décisionnelle, mais, contrairement à Black et Amina, leurs rédacteurs en chef donnaient des indications

${ }^{28} \mathrm{P}$. Dévoluy, ancien de Radio Monte Carlo, directeur adjoint d'Africa $N^{\circ} 1$ à partir de 1994.

${ }^{29}$ Parmi lesquels J-P. Guetny, ancien enseignant coopérant au Togo qui dirige aujourd'hui Le Monde des Religions et a été expert pour le Rapporteur spécial de l'ONU sur le racisme et pour le Conseil de l'Europe. ${ }^{30} \mathrm{~A}$. Bah Diallo, veuve d'un journaliste de $J A$ opposant au président guinéen Sékou Touré. 
exigeantes, qui garantissaient une ligne éditoriale de qualité.

Les espaces de collaboration étaient rares. À l' $A I T V$, il y avait une conférence de rédaction quotidienne ${ }^{31}$ et les intermittents disposaient de la salle de réunion où trônait le fil $A F P^{32}$. À $J A E$, les conférences de rédaction se tenaient dans le bureau du rédacteur en chef blanc qui accueillait une jeune diplômée blanche issue de Sciences Po bénéficiant des prérogatives d'une «star» métropolitaine en charge de la coopération française, une secrétaire de rédaction préretraitée aux préjugés coloniaux, et moimême, salariée multitâches. Les journalistes pointaient ${ }^{33}$, «pissaient de la copie $»^{34}$, des publireportages $^{35}$, ou traitaient des données fournies par les banques ${ }^{36}$.

Les piges, sans encadrement, sans équipement ni financement, sont souvent la seule perspective de carrière ${ }^{37}$. Quand je courais les piges ${ }^{38}$, pour sortir de mon isolement, j'ai adhéré au Club de la Presse Africaine à Paris et à l'Association des Journalistes de la Presse Étrangère au Sénégal qui organisaient des interviews collectives et facilitaient l'accès aux personnalités. La

${ }^{31}$ Dirigée par J-L. Maertens, responsable Afrique d'EuroNews.

${ }^{32}$ Service d'informations en temps réel de l'Agence France-Presse; c'est la seule rédaction qui en disposait.

${ }^{33}$ Ils devaient indiquer à la secrétaire la raison de leurs allées et venues.

${ }^{34}$ Expression péjorative qui désigne le travail des pigistes qui produisent des articles en quantité au détriment de la qualité. À $J A E$, les salariés devaient être « rentables » et noircissaient le plus de feuillets (un feuillet égal 25 lignes de 60 caractères, soit 1500 signes) possibles par numéro.

${ }^{35} \mathrm{Ou}$ publirédactionnel: information commanditée par un client. La loi impose que son caractère publicitaire soit explicite, mais certains médias font croire à une information indépendante.

${ }^{36} J A E$ publiait un numéro spécial annuel consacré au classement des banques privées de la zone CFA.

${ }^{37} \mathrm{La}$ plupart parviennent difficilement à « exercer en tant que journalistes », quelques éditorialistes «font carrière », sans être salariés d'une rédaction.

${ }^{38}$ Quand un journaliste n'est pas salarié d'un seul support, c'est ainsi que l'on qualifie le fait de proposer des piges à plusieurs magazines plupart des médias versent des droits d'auteur et l'isolement va de pair avec l'instabilité financière. Correspondante à Dakar, mes honoraires étaient régulièrement versés par Le Moniteur, mais il fallait menacer Africa $N^{\circ} 1$ de démission pour être payée.

En l'absence de collectif de travail, les individus sont isolés et mis en concurrence, sans pouvoir s'unir pour obtenir de meilleures conditions de travail et de formation (Linhart, 2009). Cette atomisation n'empêche pas les stratégies d'acteurs. J'ai souvent intégré les rédactions sur recommandations, comme à Élite International, où j'étais salariée, impliquée dans la chaîne de fabrication et dotée d'une carte de presse. Pas de collectif de travail dans les bureaux parisiens d'Eurafripub ${ }^{39}$, mais le correspondant d'Africa $N^{\circ} 1 \mathrm{~m}$ 'a formée à la radio pour le remplacer. Et pour le direct du Journal des Auditeurs, je collaborais avec un monteur sénégalais qui a présenté cette quotidienne après mon départ. Les correspondants parisiens d'Africa $N^{\circ} 1$ - le français R. Minangoy ${ }^{40}$, le béninois $M$. Yousoufou et moi-même -, bénéficiaient du prestige de «la» radio africaine et d'une apparente liberté d'expression.

Ces rédactions étaient instables. Après le décès du secrétaire général camerounais ${ }^{41}$ de $J A E$, qui m'avait engagée pour équilibrer les responsabilités éditoriales, j’ai été licenciée $e^{42}$, à la demande de $J A$, Jean-

\footnotetext{
39Depuis 1992, Eurafripub (Médias d'Afrique depuis 2002), détenue par Elf, Jean Lefebure/Vinci, Comilog/Gabon, la Sofirad et Africa $N^{\circ} 1$, exploite la radio en France et fournit des équipements techniques aux collaborateurs.

${ }^{40}$ Lors de son "africanisation ", Africa $N^{\circ} 1$ a congédié cet ancien correspondant de RFI en Côte d'Ivoire. R. Minangoy a ensuite dirigé son bureau parisien pour la Sofirad.

${ }^{41}$ En moins d'un an, deux rédacteurs en chef métropolitains (J-P. Béjot et R. Sadoux) et deux secrétaires généraux camerounais ( $\mathrm{P}$. Essomba et $\mathrm{J}-\mathrm{V}$. Tchienehom) se sont succédés à $J A E$.

${ }^{42}$ Après trois ans de procédure aux Prud'hommes, j'ai obtenu des dommages et intérêts pour licenciement abusif, en même temps que deux autres journalistes.
} 
Baptiste Placca ${ }^{43}$, plume gabonaise réputée, qui rejoignait Gideppe comme rédacteur en chef avec sa propre équipe. Informé de mes relations politiques au Sénégal ${ }^{44}$, B-P. Talla m'a offert de réintégrer mon poste ${ }^{45}$. En dépit de mes précédents reportages pour ce titre, Africa International, m'a alors commandé un papier, en se réservant le droit de le refuser.

Une féroce concurrence règne: combien de projets conçus collectivement ont été présentés aux bailleurs sans m'en informer ? Combien de mes idées de papiers, publiées par d'autres rédacteurs? À $J A E$, face aux plumes reconnues et à l'agenda "promotionnel », il était difficile de soumettre des sujets originaux. À RFI, selon Hugeux, «Il suffit [...] d'entendre ce que les uns disaient sur les autres, de traverser la salle de rédaction, de sentir la haine palpable qui peut diviser cette rédaction, pour mesurer la réalité du phénomène» (Anonyme, 2007). J'ai connu une atmosphère semblable à l'AITV : tout en me félicitant pour mon travail à «la» radio africaine, on feignait d'« oublier » que j'avais collaboré avec l'AITV avant mon départ en Afrique, pour m'assigner des tâches de débutant. Ces médias, qui laissent espérer l'émergence d'une information spécifique des minoritaires, restreignent ainsi leur expression.

\section{Reproduction du déséquilibre Nord/Sud dans la production de l'information}

Pouvoir et savoir sont inséparables : le maintien de liens étroits avec l'excolonisateur pèse sur la production de l'information. Les sujets des correspondants sont transmis par valise diplomatique ou enregistrés par téléphone. À l'AITV, dans le droit fil de l'hégémonie de l'information

\footnotetext{
${ }^{43}$ Aujourd'hui chroniqueur sur RFI et de retour à $J A$.

${ }^{44}$ Membres du gouvernement, députés.

${ }^{45} \mathrm{Ma}$ prétendue insuffisance professionnelle n'avait plus lieu d'être.
}

occidentale $^{46}$, les contributions des correspondants locaux étaient considérées comme de la matière première et montées comme des images d'illustration. À Africa $N^{\circ} 1$, on interrompait l'enregistrement de mes papiers critiques sur la coopération française. Le «concours» des Africains à Multiplex se résumait aux brèves " pays » du bulletin, qui assurait la promotion de Radio France et présentait quelques médias francophones. Selon Perret (2010), RFI réussit à concilier le point de vue français, les luttes politiques africaines et la coopération franco-africaine. Mais d'après Hugeux, «le statut d'envoyé spécial et de correspondant de RFI en Afrique est tout sauf une sinécure. On n'est plus le notable de la "Voix de la France". [...] On est quelqu'un qui est, en permanence, suspecté de rouler pour l'Autre » (Anonyme, 2007). Pour ma part, j'étais sollicitée par les cabinets ministériels, les opposants, la rébellion casamançaise ${ }^{47}$ et les réfugiés mauritaniens ${ }^{48}$.

Certains médias réclament des titres engagés, d'autres des papiers neutres et les pseudonymes sont courants pour les opinions divergentes. Les experts monopolisent la mise en perspective des faits et les reportages qui impliquent des dépenses. J'ai financé moi-même mes reportages à Dakar et à Paris pour Africa International, ainsi que mes papiers sur le coup d'État militaire gambien ${ }^{49}$ et sur le deuxième festival international de jazz de Saint-Louis du Sénégal pour Africa $N^{\circ} 1$. Les pigistes réalisent une veille permanente avec tout ce qui peut faire office de dépêche d'agence. À Dakar, j'achetais à mes frais la

\footnotetext{
${ }^{46}$ Déséquilibre mis en évidence en 1980 par le rapport MacBride qui a causé le retrait de l'UNESCO des États-Unis et de la Grande-Bretagne.

${ }^{47}$ Pendant une vingtaine d'années un conflit armé a opposé le Mouvement des Forces Démocratiques de Casamance et le gouvernement sénégalais. RFI n'en a parlé que lors de l'enlèvement de touristes français en 1995.

48De 1989 à 1991, un conflit entre le Sénégal et la Mauritanie a provoqué l'exil de centaines de milliers de réfugiés Noirs au Sénégal et Maures en Mauritanie. ${ }^{49} \mathrm{La}$ Gambie est enclavée dans le territoire sénégalais.
} 
presse officielle et privée et je m'appuyais sur les radios privées Sud Radio et Walfadjri. Cette organisation pèse sur les décisions rédactionnelles qui restent le domaine réservé des responsables éditoriaux et transparait dans les contenus qui perpétuent les régimes discursifs néocoloniaux.

\section{Des rapports de pouvoir genrés et ethnicisés}

Mon propre parcours, ainsi qu'une enquête de terrain auprès de femmes noires diplômées travaillant dans la presse, contribuent à montrer que les médias reproduisent la division genrée du travail domestique et professionnel. Comme dans les autres secteurs professionnels, les inégalités dans les salaires et les catégories professionnelles y persistent (Maruani, 1998). En regardant comment les catégories émergent des témoignages ${ }^{50}$ sur les parcours professionnels, je tente une analyse intersectionnelle (Bilge, 2009b) des conditions qui exacerbent ou neutralisent les privilèges et les désavantages (Browne et Misra, 2003). Mon hypothèse est que les sujets mettent en place des stratégies discursives qui donnent plus ou moins de force aux catégories sociales auxquelles on les renvoie afin de résister aux assignations.

Dans la presse, à ma connaissance, seules trois femmes noires ont créé un titre et/ou assuré la responsabilité éditoriale et/ou managériale d'un support ethnique panafricain francophone - M-R Biloa $^{51}$, M-J. Serbin, et moi-même - : nos carrières sont inhabituelles. Celles qui ont réussi à se faire une place au soleil des grands médias audiovisuels mainstream font figure de modèle à suivre. Ainsi, la Martiniquaise Audrey Pulvar, qui a d'abord présenté le JT aux côtés d'un Blanc, se souvient: "Quand je suis arrivée pour le

\footnotetext{
${ }^{50}$ Enquête réalisée en 2008/2009 auprès d'une dizaine de sujets pour mon mémoire de M2 recherche en psychodynamique du travail.

${ }^{51} \mathrm{La}$ seule à ne pas diriger un féminin (Africa International).
}

Soir 3, il a eu [...] tous les jours des interviews "première femme noire" » [...]. Je n'arrêtais pas de répéter que vous pouvez mettre toutes les Audrey Pulvar et tous les Harry Roselmack que vous voulez à la télé, c'est pas ça qui va faire en sorte que le mec qui est noir $[. .$.$] va trouver plus facilement un$ appartement ou un boulot $[\ldots]$. On va s'habituer au fait qu'un Noir qui arrive sur une manifestation n'est pas forcément, ni du service d'ordre, ni un "sans papiers", mais peut être aussi un journaliste de TF1...» (Garcia, 2009). Cette autre journaliste martiniquaise raconte: Je devais présenter le JT en Guadeloupe. Quand je suis arrivée, le directeur a eu un mowvement de recul et il me dit: "on a changé d'organisation". Il m'a proposé de présenter les programmes. D'être speakerine quoi! Je l'ai pris comme du racisme! Comme les jeunes des quartiers populaires aujourd'hui, leur sentiment d'être racialisée l'emporte sur leur conscience des inégalités genrées.

Audrey Pulvar n'a pas échappé à la " fabrique de l'ethnique » (Lada, 2004). "Quand [Nicolas Sarkozy] me dit "je sais que ce sujet [les reconduites à la frontière, NDR] vous touche" [...], quand je [lui] dis "en tant qu'être humain", je lui démontre que mon interview n'est pas partisane, parce que si c'était une militante [...], je l'aurais laissé s'enfoncer! Je [...] lui aurais dit "mais en tant que quoi? En tant que femme? En tant que Noire?" »(Boublil, 2009). Son interlocuteur l'assigne à une ethnicité politiquement engagée et elle est consciente des autres catégories auxquelles elle est associée. En France, quand vous êtes noire, on a du mal à imaginer que vous puissiez. obtenir un poste de représentation ou de responsabilités. On nous cantonne à des sujets précis. Au début, je pouvais rencontrer des auteurs européens, américains. Et puis à la fin, c'était uniquement l'Afrique ou les Noirs! J'ai compris que je n'évoluerai pas à cause de ma couleur de peau. Chaque fois qu'il y avait des postes où on devait intégrer des journalistes, curieusement, personne ne savait de qui ça dépendait. Et j'ai décidé de partir, car ils ont intégré une fille arrivée deux ans après moi. J'ai trouvé ça choquant. Étant une des premières journalistes 
noires de la station, j'avais l'impression d'être devenue une caution. Cette amie au service anglais, a réussi à se faire intégrer, mais elle a jamais pu monter dans la biérarchie. Quand j'ai annoncé que je partais, c'étaient des lamentations à n'en plus finir. Je ne savais pas que jeétais si importante pour eux !' raconte une ancienne productrice martiniquaise à RFI. Cette Afro-antillaise choisit une stratégie d'évitement: J'ai jamais cherché à avoir un travail dans une société caucasienne. Là où je travaillais, tout le monde était noir, donc il y avait pas ce problème-là ! En fait, dans ce milieu, c'était plutôt positif. Si j'avais été blanche? Je ne serais jamais allée dans un milieu noir. Pourquoi? Travailler dans un milieu noir, sur des questions et des problèmes de Noirs... Au moins, j'étais avec des gens avec qui on pouvait se comprendre! Or, parmi leurs "semblables", elles restent soumises aux discriminations dues au sexe et à l'âge et sont précarisées. Je fais un métier où, en France en 2008, rares sont les CDI et il faut s'accommoder de cette situation, explique cette jeune pigiste. C'est plus douloureux à cinquante ans, selon les confidences de cette ex-productrice radio: J'ai toujours été à l'affût d'opportunités. J'essaie de me battre. C'est de plus en plus difficile, je fais des consultations ou quelques papiers en freelance pour essayer de vivre... De surcroit, ces expériences sont stigmatisées par les employeurs de l'Hexagone et mes brèves années de pratique journalistique en Afrique ont totalement éclipsé quinze années d'expérience en France.

Ainsi, pour échapper aux discriminations dues à leur sexe et/ou à leur couleur de peau et accéder aux carrières qu'elles estiment mériter, certaines créent une entreprise, comme cette assistante de presse : Je ne vais pas aller me battre pour dire que, parce que je suis femme, ou parce que je suis noire, j'ai réussi à faire ci, malgré que tout le monde était contre moi! Finalement, j'ai décidé de créer une société. J'étais assistante de direction: je me suis juste coulée dans quelque chose qui était fait! J'ai dit : "je suis capable de le faire toute seule. Je vais faire ma petite régie pub". Pour ne plus avoir de patron sur le dos! Je pouvais travailler comme je voulais. Je ne vois pas d'ambition là dedans. C'était faisable! Les quelques femmes noires qui réussissaient, elles s'en sortaient toutes seules! Par exemple, Marie Jeanne [Serbin] qui avait fait Brune, Marie Roger Biloa... Elles étaient salariées, mais elles ont fait leur propre truc! Elles sont pas restées là-dessous... Marie Roger a commencé comme secrétaire à $J A^{52}$, et puis elle est montée, parce qu'elle en a eu marre! Et pour monter, je pense qu'il faut créer soi-même son truc! Parce que même si je suis à un poste de directrice machin tralala, il y a toujours un chef pardessus! Pas vraiment indépendant! Elle associe sexe et race à la subalternité et dévoile un niveau d'aspiration différent des stéréotypes.

La couverture de l'actualité demande une flexibilité qui révèle l'interrelation entre travail professionnel et sphère privée. Jeune mère à $J A E$, j'ai soudain vu les conférences de rédaction convoquées à 19 heures, les papiers commandés in extremis et les reportages à l'étranger se multiplier et, à l'AITV, je devais parfois emmener ma fille en salle de montage le dimanche. Si la vie familiale des femmes s'invite souvent dans leur vie professionnelle, le salaire révèle la valeur du travailleur dans la hiérarchie. Pour cette jeune pigiste: Même si ça ne me rapporte pas d'argent, pratiquer le journalisme, c'est plus une mission qu'un métier. Elle a intériorisé l'injonction genrée au travail non rémunéré. Les titres professionnels reflètent aussi l'assignation à des fonctions "féminines »: dans l'ours ${ }^{53}$ d'Élite Madame, j'apparaissais comme coordinatrice de la rédaction et mon homologue blanc, qui exerçait les mêmes fonctions, comme directeur de l'information.

Les discriminations et les inégalités dont font l'objet ces femmes reflètent l'imbrication des rapports sociaux de genre, de race, de classe et d'âge. Des enquêtes menées aux États-Unis sur le marché du travail ont montré l'intersectionnalité de la race et du sexe et constatent que certaines

\footnotetext{
${ }^{52} \mathrm{Si}$ cette rumeur s'avérait vraie, son parcours serait d'autant plus emblématique.

${ }^{53} \mathrm{~L}$ '《 ours » est un encadré, en début ou en fin de journal, qui contient les mentions légales obligatoires - éditeur, directeur de publication, collaborateurs, nom et adresse de l’imprimeur, dépôt légal, ISSN.
} 
conditions peuvent rendre plus saillante l'une ou l'autre de ces catégories sociales (Browne et Misra, 2003). Par ailleurs, une étude comparative franco-brésilienne des rapports entre catégories et trajectoire montre l'ordre d'importance de l'âge, de l'instruction, du sexe et de la race (Araujo Guimaraes et al., 2008). Cela se vérifie sur le terrain: De toute façon, l'âge fait partie des critères qui font que l'on balance votre truc à la poubelle. Je peux même rajouter le fait que je sois bien joufflue et plus que ronde... explique cette quinquagénaire pour laquelle l'apparence physique n'est pas anodine. Pour cette journaliste de 32 ans : C'est le fait d'être jeune, parce que ceux qui ont la côte dans l'audiovisuel, c'est les quadras français, indiens, blancs, noirs. Et moi, je suis en plein dans la case des trentenaires, où tout, sexe, race, études, c'est la monise. Consciente de l'interaction des catégories sociales, elle neutralise sexe et race, et présente l'âge comme source des privilèges ou des désavantages. Elle s'attarde aussi sur l'effet du contexte qu'ont démontré les recherches nord-américaines (Browne, Misra, 2003). On me demande: "Avant, t'as bossé dans quelle boîte? "Parce que c'est des rédacteurs en chef de 35 ans, 40 ans qui ont connu des Blacks, des Arabes, dans leur cour de récréation. C'est différent, parce qu'on est dans un milieu particulier, on est dans l'audiovisuel. Si j'avais parlé de France 3 Chateauroux, on de France 3 Dijon, je ne dirais peut-être pas la même chose. On est à Paris : les gens voyagent, ils ont des copains musiciens, artistes africains, indiens, chinois... Les problèmes qu'un journaliste noir peut rencontrer dans le métier, c'est par exemple, quand j'ai besoin de trouver un témoin à Châteauroux... Soucieuse de ne pas attribuer toutes ses difficultés au racisme, cette jeune pigiste reconnait néanmoins qu'il lui arrive d'être renvoyée à son origine ethnique et que l'addition de l'âge, du sexe et de la race exacerbe les discriminations : Quand tu débarques d'Alsace dans une grosse boîte de production à Paris et que ta chef, c'est une Black, je pense que ça a dûu lui faire quelque chose à mon stagiaire !! Il me reconnaît comme supérieure biérarchique quand il a chand aux fesses! Il aura moins le trac vis à vis de moi que d'une femme blanche! Ca renvoie à l'inconscient collectif qui fait que les Noirs, on les prend pour des gens qui ne sont pas capables, des gens inférieurs! Avant de voir la coordinatrice, il va voir une "Re-nol" ! Quand elle travaille pour des médias mainstream, elle associe parfois sa professionnalité à un engagement militant: Ne rien laisser passer. Je lui dis: "Attends, comment ça "la petite Blackette?? Déjà 'petit', c'est réducteur! Le suffixe 'ette', je ne t'en parle pas!" Donc stop quoi! Tu dis 'une jeune Afro-américaine on une Black.' Mais 'une petite Blackette'...!

Les sujets ont tendance à naturaliser les discriminations dues au sexe ou à l'âge : j'ai toujours été femme; je suis jeune, j'ai des choses à apprendre; il y a des choses que je fais plus lentement; à mon âge, c'est plus difficile d'être manipulée. Cette intériorisation de catégories essentialisées est telle que, pour cette assistante afro-caribéenne, face à la concurrence des hommes sur le marché du travail, le sexe est un avantage : ce que je faisais - secrétariat, assistanat - c'est un boulot de femme! Les marqueurs sociaux, comme l'accent, le nom ou le patronyme, peuvent privilégier ou pénaliser la même personne. Quand les gens me voient, ils sont étonnés, parce que j'ai pas d'accent. On ne peut pas savoir, sauf par mon nom [nom et prénom africains]. Ils s'attendent à voir une Japonaise... Certaines adoptent des stratégies "patronymiques»: Je n'utilise pas mon nom de femme mariée [prénom chrétien et nom juif]. On peut se dire: "elle est vietnamienne on africaine".

La relation à l'ethnicité varie selon que l'on appartient à un groupe dominant ou dominé (Juteau, 1999). Ainsi, certains sujets issues des oligarchies locales s'appuient sur leurs références de classe, comme cette journaliste et écrivaine afro-martiniquaise. Ils s'attendaient à voir quelqu'un avec qui ils auraient pu faire tranquillement du paternalisme. Ils voyaient qu'ils pouvaient pas me mettre en situation d'infériorité. On va au restaurant, "ah, c'est quoi ça?". "C'est un couteau à poisson". Venant de moi, c'est vexant. Vous avez les mêmes références, vous les connaissez parfaitement... Tout Blancs qu'ils soient, je n'ai rien à leur envier. Parce que pour eux, le Noir, c'est la personne de basse 
extraction qui n'arrivera jamais à leur niveau : le pawre paysan ou le balayeur ou la fille de salle. Et ils ont jamais vu de Noirs ingénieurs, médecins, physiciens... Noir, ça doit correspondre à la représentation. Les Noirs, ils sont là pour qu'on les aide et moi j'entre pas dans cette représentation. Quand ça va jusqu'à "doudou" et "cheveux de mouton", j'essaye par l'bumour de les remettre à leur place. Je préfere qu'on apprécie mes compétences et non pas ma façon d'être ou ma couleur. Son identité de classe minore l'expérience d'être racisée, le sexe et l'âge. Cette autre ancienne journaliste, issue selon ses propres termes du «sérail intellectuel et politique martiniquais », n'explique les discriminations que par son âge et sa formation. En Martinique, je recevais beaucoup de compliments pour mes articles qui avaient un plus grand retentissement que tout ce qu'ils [les collègues hommes] pouvaient écrive on faire! Mais il eut fallu que j'aie fait des études de journalisme pour pouvoir faire une carrière sérieuse... En tout cas, c'est ce qu'on m'a dit... Il y en avait qui n'avaient pas de diplômes du tout! Alors j'ai toujours pensé que c'était mes diplômes, plus variés et plus élevés, plutôt que mon sexe! Je crois que surtout j'étais encore trop jeune pour pouvoir rester là-dedans ! J'avais pas le cour assez tanné! Je ne pense jamais spontanément: "c'est parce que je suis une femme", "c'est parce que je suis noire". Je suis très critique à propos de la discrimination positive. C'est une connerie absolue, une solution de facilité! Parce que nous avons les moyens de réussir sans mettre en avant la couleur de peau! Je ne veux pas être une victime! Je veux être une personne qui choisit son destin! Elle ne se pense pas comme dominée, car, issue de la classe dominante, elle est incapable d'assumer les représentations négatives de la différence et donc de dénoncer les discriminations (Poli, 2004). L'habitus ${ }^{54}$ (Bourdieu, 1979) de ces " filles de », des classes oligarchiques, les autorise à partager les codes des classes dominantes blanches, à évoluer dans leur monde social et les aident à résister aux atteintes dévalorisantes. Cet habitus influe aussi sur la pratique professionnelle. Ainsi, c'est sous pseudonyme que j'écrivais ma

\footnotetext{
${ }^{54}$ Acquis sociaux incorporés au cours de la
} socialisation. chronique santé pour Amina, non pour m'exprimer sans entraves, mais pour camoufler une production journalistique purement alimentaire dans une revue populaire. En effet, née dans une famille d'intellectuels, je ne me suis jamais sentie concernée par l'émancipation féminine «de masse ${ }^{55}$ prônée par ce magazine féminin. Une posture proche de celle de M-J Serbin, qui oppose la «Rolls» Brune à la «2CV»Amina (Fele, 2007), une comparaison imagée qui rend clairement compte d'une distinction de classe.

En vertu de la division sexuée et racisée du travail, les Noires sont le plus souvent vouées au rôle de présentatrice ou d'assistante, ou, quand elles sont journalistes, aux piges pour les colonnes féminines ou sociales et aux sujets "sexy $»^{56}$. Elles sont renvoyées à leur condition genrée: lors d'une conférence de presse à l'ambassade de France, à une question sur la Francophonie, un ministre français s'est contenté de me répondre: Madame, vous êtes très belle. À Black, une femme noire sans visage, le pubis arborant les couleurs du drapeau français, illustrait en couverture l'un de mes articles sur le racisme envers les Noires. Je fus ensuite convoquée au domicile de l'éditeur pour toucher mon chèque, qu'il me remit... vêtu d'un peignoir de bain !!! Les femmes noires sont essentialisées comme des objets corporels à la sexualité licencieuse ou passive (hooks, 2008). Dans la littérature coloniale, l'Africaine est inscrite dans la domesticité et la vénalité (Jeurissen, 2003). Dans la culture populaire, les représentations dégradantes des corps noirs féminins sont hantées par l'esclavage (Hill Collins, 2004) dans le contexte de la domination masculine

\footnotetext{
${ }^{55} \mathrm{La}$ pérennité du premier mensuel féminin africain repose sur sa politique de proximité. Ses lectrices - de la vendeuse de beignets du coin de la rue à la première dame de la république - sont friandes de publicités pour crèmes éclaircissantes et défrisantes et de son roman-photo, qui en font un magazine bas de gamme très populaire.

${ }^{56}$ Se dit des papiers qui font vendre, comme la contraception des adolescentes sénégalaises ou la prostitution des femmes noires à Paris.
} 
postcoloniale (Le Bihan, 2007 ; 2011). Cette administrative guadeloupéenne de trentecinq ans en est bien consciente: L'image qu'on a de la femme noire, c'est qu'elle est bonne à baiser. Parce qu'on est traitées comme des prostituées! Je le vis très mal. C'est le travail ou la baise! Ca n'est pas fini... Approches frontales, harcèlement sexuel, grossièreté, insinuations rendent compte de l'intersectionnalité du sexe et de la race, qui entraine une hypersexualisation. La femme noire a une certaine réputation. [Le directeur NDR] nous faisait savoir que les femmes africaines, elles sont faciles! Tant que tu as de l'argent, ça y va!, s'indigne cette aide comptable. Mais là encore, la classe trouble le sexe et la race: elle peut neutraliser l'assignation à une disponibilité sexuelle permanente. Dans les relations professionnelles, l'bomme blanc, face à une femme noire qui, clairement, est d'un milieu social plus élevé que le sien, avec une certaine distance, ne va pas se sentir en posture de séduction, parce qu'il ne va pas contrôler le double handicap. Elle est femme, noire, mais surtout elle a un statut social qui permet pas... Ils ont pas les codes, donc ils sont moins à l'aise. Avec des femmes dont ils ont une image infériorisée, ils savent comment maitriser les choses. C'est le maître blanc qui décide, le droit de cuissage! C'est eux qui décident que cette femme leur est inférieure. Ça peut être une fille de niveau social supérieur, mais s'il a décidé qu'il est le maître... Cette journaliste-écrivaine de 56 ans se contredit, montrant que, dans les interactions des catégories et la dynamique des privilèges et des désavantages (Browne, Misra, 2003), la racialisation peut aussi effacer la classe (Kergoat, 2009). Le stéréotype des femmes de couleur reproduit la dichotomie entre la maman et la putain, entre "l'africanité des vrais mamas aux formes généreuses et au teint foncé » et la beauté noire conforme aux canons européens (Jeurissen, 2003), associée à une sexualité pathologique qui appelle le harcèlement (Poiret, 2005). Dans les rédactions, je souffre moins que la blonde. C'était elle qui était victime de discrimination. On va lui parler de sexe sans lui demander son avis! Tu ne vas pas me regarder comme ça, ou me demander des choses que tu demandes à la blonde. Parce que je suis là pour le boulot! explique cette journaliste de 32 ans.
Son teint foncé, son grand nez busqué aux narines dilatées, sa bouche charnue, ses hanches larges et ses cuisses épanouies ne l'incluent que de façon marginale dans les critères de la «beauté noire» occidentalisée. Elle se sent peu érotisée et nie les avances à la fois pour neutraliser les stéréotypes hypersexuels et pour respecter les codes professionnels.

Dans les médias minoritaires, il apparait que les femmes noires doivent faire face aux assignations sociales ${ }^{57}$ issues des formes multiples et enchevêtrées de domination. Leurs témoignages sur leur parcours professionnel rendent compte d'une construction performative de leur subjectivité en marge de la norme assignée. Si «dire, c'est faire », ces discours sont des stratégies discursives qui dépassent les représentations imposées en minorant leur expérience de sujets genrés, ethnicisés, niés comme sujet de discours. Les sujets rencontrés au cours de l'enquête deviennent ainsi des sujets autonomes qui résistent à l'assujettissement ${ }^{58}$.

\section{Fonction reproductive des médias francophones panafricains et éthique journalistique}

Comment expliquer que les médias reproduisent le système néocolonial de domination ? Pour Godelier (1984), les dominés consentent à leur domination. Mathieu (1985), pour sa part, avance que « céder n'est pas consentir»: la violence symbolique de la domination provoquant une conscience fragmentée qui limite les possibilités d'action. Quelle que soit la dialectique de la reproduction des schémas de pensée du Nord dans les médias minoritaires, pour E. Fall, rédacteur en chef adjoint, la rubrique Sports était, in petto, le seul espace de liberté à $J A$. À Élite

${ }^{57}$ Pour n'en citer que quelques unes : l'invisibilité et le silence de la sphère domestique et privée, le travail subalterne, non ou peu qualifié, etc.

${ }^{58}$ Analyse inspirée de la lecture croisée de Foucault et Butler réalisée par Sanna (2006). 
International, il fallait taire les dysfonctionnements d'Air Afrique et la publication des «dossiers pays » de $J A E$ était soumise à validation politique. L'éthique de ces médias repose, en principe, sur leur mission vis-à-vis des minoritaires : rendre compte des « effets néfastes du régime de Sékou Touré [...], des dérapages de Mobutu $^{59}$ et de Bokassa ${ }^{60}$, [...] faire comprendre aux Africains que Kadhafi ${ }^{61}$, c'est une fumisterie. [...] Au Mali, beaucoup pensent que nous avons aidé à faire tomber Moussa Traoré ${ }^{62}$ en 1991. [...] J'ai incité [Amadou Toumani Touré ${ }^{63}$ à ne pas revenir tout de suite [...].» (Boisbouvier, 2010, [3/5]). Pour Hugeux, il y a confusion des genres : les médias n’ont pas à conseiller les gouvernants : «Il y a [...] des pratiques [...] totalement indéfendables - par exemple, de monnayer un titre de couverture plus ou moins bienveillant, [...] alors qu'on est payé pour faire du reportage »(Anonyme, 2007). À quoi le journaliste congolais Baudoin Amba Wetshi (2008) renchérit à propos d'un reportage de vingt-quatre pages publié dans Afrique Magazine ${ }^{64}$ sur la République démocratique du Congo. «Certains passages de ces 'papiers' dégagent, au mieux, une paresse intellectuelle patente, au pire, de la manipulation ». Thierry Perret critique aussi ce «mercenariat de plume», sans considérations déontologiques. «Les interdictions dont nous avons été victimes tout au long de notre histoire sont la preuve de notre indépendance. [...] Qu'on ait fait des compromis, qu'on ait tenu compte d'un certain nombre d'impératifs, bien évidemment [...]. D'après moi, il n'y a pas de journal complètement indépendant, il n'y a pas de pays complètement indépendant. L'indépendance, ce n'est pas un absolu, cela a des limites. L'essentiel est de limiter ces

\footnotetext{
${ }^{59}$ Président du Zaïre de 1965 à 1997.

${ }^{60}$ Président de la République centrafricaine de 1966 à 1976, autoproclamé empereur sous le nom de Bokassa Ier de 1976-1979.

${ }^{61}$ Propos tenus en 2010.

${ }^{62}$ À la tête du Mali de 1968 à 1991.

${ }^{63}$ Amadou Toumani Touré, président du Mali, élu en 2002, renversé en 2012.

${ }^{64}$ Groupe $J A$.
}

limites $[. .] J$.$A a sauvegardé l'essentiel de$ son indépendance. [...] Mais Jeune Afrique coexiste avec des pays et des gouvernements qui ont beaucoup de pouvoir, et il est obligé de composer. Et les hommes du pouvoir, eux aussi, composent avec nous. [...] il y a une espèce de négociation. Quand on disait à Mobutu: "Jeune Afrique a dit», il répondait: "Foutez-moi la paix avec Jeune Afrique! Ce n'est pas la Bible! Cela signifie bien, tout de même, que ce journal est crédible et qu'il faut, par conséquent, tenir compte de ce qu'il écrit. [...] Vous êtes [...] obligés de vous accommoder de pouvoirs [...] auxquels il n'y a pas d'alternative. [...] Que faites-vous dans un continent où toute l'économie appartient à l'État [...] ? [...] Vous êtes obligé de tenir compte des limites que vous ne pouvez pas dépasser [...] [Le journal évite] de donner une information fausse dont vous savez pertinemment qu'elle est fausse ou complaisante. [...] Dire le maximum sans tout dire. [...] Il y a des limites évidentes. On peut très bien les dépasser, mais, le lendemain, il n'y a plus de Jeune Afrique en Tunisie. [...] Mais est-ce bien pour les lecteurs tunisiens ? [...] La contrainte commerciale ne doit pas conduire à falsifier la vérité. [...] Vous ne trouverez pas dans Jeune Afrique quelque chose qui soit contraire à la vérité. Il n'y a pas toute la vérité, mais il n'y a rien de contraire à la vérité. C'est la ligne jaune de l'information. [...] Regardez RFI, vous avez les mêmes problèmes. » (Boisbouvier, 2010, [4/5]). Conçus comme des instruments de libération, les contraintes politiques, économiques et stratégiques auxquelles sont soumis les médias panafricains francophones les transforment en outils de domination.

\section{Conclusion}

Parameswaran (2008) a montré que les médias indiens constituent un espace privilégié pour comprendre les implications de la mondialisation. Sa démarche autoréflexive rend compte des positionnalités et des implications d'une recherche réalisée par des non-occidentaux 
dans leurs propres cultures (2001). Elle explore également les représentations globalisées de la féminité, de la masculinité et de la race qui font écho aux discours coloniaux sur l'altérité (2002a; 2002b). La «glocalisation» ${ }^{65}$ a des effets sur les décisions éthiques des journalistes du Sud, qui doivent arbitrer les enjeux déontologiques au niveau local (Wasserman, Rao, 2008). Ainsi, face aux politiques d'ajustement structurel, la presse privée nigériane a servi les intérêts de l'élite commerciale locale plutôt que ceux de la société civile (Hall, 2012). Brisset-Foucault (2009) analyse les modèles médiatiques africains en se basant sur l'hétérogénéité des mobilisations informationnelles nationales qui, pour s'internationaliser, doivent répondre à une injonction d'«utilité sociale », d'africanisation, d'individualisation critique et d'antagonisme "soutenable » avec les gouvernements africains. Les médias minoritaires expérimentent des conditions de production qui vont de pair avec un nonrespect de la norme déontologique. En effet, leur ancrage social facilite, rend possible et limite à la fois la production et la diffusion de leurs informations.

Néanmoins, il n'apparaît pas que, contrairement aux médias dominants, les médias minoritaires prennent davantage en compte l'imbrication des systèmes de domination de genre, de classe et de race. L'enquête réalisée auprès de travailleuses noires de ce secteur professionnel m'a permis d'analyser «ce qui ne se dit pas collectivement». Leur représentation dans l'espace public et les médias minoritaires reste largement stéréotypée. Du fait de leur instruction et parfois de leur classe sociale d'origine, elles ne sont pas cantonnées dans les strates inférieures du marché du travail, mais subissent une oppression de nature raciste, sexiste et classiste. Si la race peut faire l'objet d'un déni et la classe être avancée par les sujets eux-mêmes, le genre reste le plus souvent occulté comme facteur explicatif de leur parcours professionnel. Les médias minoritaires apparaissent alors comme des niches où elles peuvent se «réfugier» pour faire face aux assignations raciales, pour produire leur propre discours sur leurs identités et participer, dans le champ journalistique, à la citoyenneté et à la sphère publique. Mais on peut aussi qualifier ces milieux de ghettos où sont relégué(e)s à la fois les travailleurs(euses), leur culture et leurs préoccupations spécifiques.

Carmen Diop

Doctorante en sciences de l'éducation

Paris 13 - EXPERICE carmendiop@yahoo.com

\section{Bibliographie}

Anonyme (2007) Les sorciers blancs et la politique africaine de la France (Interview de Vincent Hugeux, 21/05/2007), couper, copier, coller. Dans les coulisses de la guerre médiatique [en ligne, réf. du 28/06/2012] URL :

$<$ http://coupercoller.wordpress.com/2007/ 05/21/les-sorciers-blancs-et-la-politiqueafricaine-de-la-france/ $>$.

Anonyme (2012) L'bistoire du magazine Amina, Amina.mag.com [en ligne, réf. du 06/12/2012] URL: <http://www.aminamag.com/presentation du magazine.html>. Amba Wetshi, Baudouin (2008) Joseph Kabila entretient son image... à l'extérieur, 18 août 2008, [en ligne, réf. du 28/06/2012] URL :

$<$ http://losako.afrikblog.com/archives/200 8/08/18/10267659.html>.

${ }^{65}$ Combinaison des tendances «globales» et des réalités « locales ». 
Araujo Guimaraes, Nadya ; Alves de Britto, Murillo Marschner (2008) Genre, race et trajectoire: Une comparaison entre Paris et São Paulo, in Hirata, H. ; Lombardi, M.R. ; Maruani, M. (dir.) Travail et genre. Regards croisés France, Europe, Amérique latine, Paris, La Découverte, pp. 46-60.

Ardant, Philippe (1965) Le néo-colonialisme : thème, mythe et réalité, Revue française de science politique, Vol. 15, n5, pp. 837-855.

Bhabha, Homi K. (2007) Les Lieux de la culture. Une théorie postcoloniale, Paris, Payot, $334 \mathrm{p}$.

Bilge, Sirma (2009a) Théorisations féministes de l'intersectionnalité, Diogène, n²25, pp. $70-88$.

Bilge, Sirma (2009b) Smuggling Intersectionality into the Study of Masculinity, communication présentée à la Feminist Research Methods Conference, University of Stockholm.

Balandier, George (1974) Anthropo-logiques, Paris, PUF, 279 p.

Boisbouvier, Christophe (Propos recueillis par) (2010) Béchir Ben Yahmed : tout ce que vous avez toujours voulu savoir sur Jeune Afrique [1/5], Jeuneafrique.com [en ligne, réf. du 06/12/2012]

URL :

$<$ http://www.jeuneafrique.com/Articles/D ossier/ARTJAJA2598p030-039-

bis.xml0/anniversaire-france-financesarabebechir-ben-yahmed-tout-ce-que-vousavez-toujours-voulu-savoir-sur-jeuneafrique-1-5.html>

Boisbouvier, Christophe (Propos recueillis par) (2010) Béchir Ben Yahmed, l'Algérie et le Maroc [2/5], Jeuneafrique.com [en ligne, réf. du 28/06/2012] URL : $<$ http://www.jeuneafrique.com/Articles/D ossier/ARTJAWEB20101110162030/annive rsaire-algerie-france-marocbechir-benyahmed-l-algerie-et-le-maroc-2-5.html $>$.
Boisbouvier, Christophe (Propos recueillis par) (2010) Béchir Ben Yahmed et les despotes du continent [3/5], Jeuneafrique.com, [en ligne, réf. du 28/06/2012] URL : $<$ http://www.jeuneafrique.com/Articles/D ossier/ARTJA20101110161916/anniversaire -france-vietnam-terrorismebechir-benyahmed-et-les-despotes-du-continent-3$\underline{\text { 5.html }>\text {. }}$

Boisbouvier, Christophe (Propos recueillis par) (2010) Béchir Ben Yahmed: "Bien évidemment Jeune Afrique a dû faire des compromis » [4/5], Jeuneafrique.com, [en ligne, réf. du 28/06/2012] URL: <http://www.jeuneafrique.com/Articles/D ossier/ARTJA20101110160422/anniversaire -france-diplomatie-rwandabechir-benyahmed-bien-evidemment-jeune-afrique-adu-faire-des-compromis-4-5.html $>$.

Boublil, Elsa (2009) Interview d'Audrey Pulvar, in J'ai mes sources, 04/08/2009, France Inter.

Bourdieu, Pierre (1971) Le marché des biens symboliques, L'Année sociologique, Vol. 22, pp. 49-126.

Bourdieu, Pierre. (1979) La distinction : critique sociale du jugement, Paris, Minuit, $672 \mathrm{p}$.

Bourdieu, Pierre (2000) Esquisse d'une théorie de la pratique, Paris, Points, 429 p.

Brisset-Foucault, F.e (2009) Quel « autre journalisme » en Afrique? La production de modèles médiatiques africains au forum social mondial de Nairobi, Réseaux, Vol. 5-6, n¹57-158, pp. 125-156.

Browne, Irene; Misra, Joya (2003) The Intersection of Gender and Race in the Labor Market, Annual Review of Sociology, Vol. 293, pp. 487-513.

Butler, Judith (2009) Ces corps qui comptent; de la matérialité et des limites discursives du "sexe », Paris, Ed. Amsterdam, 256 p.

Butler, Judith (2004) Le Pouvoir des mots. Politique du performatif, Paris, Ed. Amsterdam, 287 p. 
Colombani, Olivier (1991) Mémoires coloniales. La fin de l'Empire français d'Afrique vue par les administrateurs coloniaux, Paris, La Découverte, 209 p.

De Lagasnerie, Geoffroy (2011) Logique de la création, Paris, Fayard, 269 p.

Delphy, Christine (1998) L'ennemi principal. I., Paris, Syllepse, 280 p.

Delphy, Christine (2001) L'ennemi principal. II., Paris, Syllepse, 388 p.

Dorlin, Elsa (dir.) (2008) Black Feminism, Anthologie du féminisme africain-américain, 1975 2000, Paris, L'Harmattan, 262 p.

Dufoix, Stéphane (2003) Les diasporas, Paris, PUF, 128 p.

Encyclopédie des Ekangs [en ligne, réf. du 5/07/2012] URL : <http://www.nkul-beticamer.org/ekang-globalrevue.php? $\mathrm{cmd}=$ repertoire $\&$ PHPSESSID $=\mathrm{h}$ 4ie6a9thghk3g9559kn9sko76>.

Fanon, Frantz (1971) Peau noire, masques blancs, Paris, Seuil, $191 \mathrm{p}$.

Fele, Safi (2007) «Brune»: le magazine féminin de la différence, 9/01/2007 [en ligne, réf. du 28/06/2012] URL : $<$ http://beaute.afrik.com/Brune-lemagazine-feminin-de-la.html>.

Garcia, Laurence (2009) Interview d'Audrey Pulvar, in Eclectik, 26/04/2009, France Inter.

Godelier, Maurice (1984) L'idéel et le matériel, Paris, Fayard, 348 p.

Guénif-Souilamas, N. (2012) Bilan AXE A: Le sujet dans la Cité : éducation, individuation, biographisation [en ligne, consulté le 27/03/2013] URL: < http://www.univparis13.fr/experice/images/stories/AXES/ A/apports axeA 2008 2011.pdf $>$.

Guillaumin, Colette (1972) L'Idéologie raciste, Paris ; La Haye, Gallimard, 378 p.

Guillaumin, Colette (1992) Sexe, race et pratique du pouvoir, Paris, Côté-Femmes, 239 p.

Guillaumin, Colette (1981) Femmes et théories de la société, Sociologie et sociétés, Vol. 13, n², pp. 19-32.
Gutiérrez Rodríguez, Encarnación (2006) Traduire la positionnalité, Transversal, $\mathrm{n}^{\circ} 6$ [en ligne, réf. du 05/07/2012] URL: $\leq$ http://eipcp.net/transversal/0606/gutierrez -rodriguez $/ \mathrm{fr} />$.

Hall, Philippa (2012) «Penser pour l'empire » au sein du Nigeria colonial et postcolonial. Presse privée, médiatrice de la politique de l'État et de l'économie mondiale, Afrique contemporaine, Vol. 4, n²40, pp. 43-60.

Haraway, Donna (1988) Situated Knowledges, Feminist Studies, Vol. 14, n³, pp. $575-599$.

Harding, Sandra (1990) Feminism, Science, and the Anti-Enlightenment Critiques, in Nicholson L.J. (ed.) Feminism/Postmodernism, New York; London, Routledge, pp. 298320.

Harding, Sandra (2003) The Feminist Standpoint Theory Reader: Intellectual and Political Controversies, New York/London, Routledge, $400 \mathrm{p}$.

Hartsock, Nancy C.M. (1998) The Feminist Standpoint Revisited and Other Essays, New York, Basic Books, 272 p.

Hill Collins, Patricia (2004) Black Sexual Politics, New York/London, Routledge, 384 p.

hooks, bell (2000) Feminist Theory: From Margin to Center, Cambridge, South End Press, 179 p.

hooks, bell (2008) Sororité : la solidarité politique entre femmes, in Dorlin, E. (éd.) Black Feminism, Paris, L'Harmattan, pp. 113134.

Jeurissen, Lissia (2003) Colonisation an masculin et mise en corps de la féminité noire, Séminaires FER-ULg 2002, 08/05/2003 [en ligne, réf. du 28/06/2012] URL : $<$ http://www.congoforum.be/upldocs/Jeur issen.pdf $>$.

Juteau, Danielle (1981) Visions partielles, visions partiales, in Sociologie et sociétés, Vol. 13, n², pp. 33-48.

Juteau, Danielle (1996) L'ethnicité comme rapport social, in Mots, Vol. 49, $\mathrm{n}^{\circ} 49$, pp. 97-105. 
Juteau, Danielle (1999) L'ethnicité et ses frontières, Montréal, Presses de l'Université de Montréal, 232 p.

Kergoat, Danièle (1998) Rapports sociaux et division du travail entre les sexes, in Maruani, M. (dir.) Les Nouvelles frontières de l'inégalité. Hommes et femmes sur le marché du travail, Paris, La Découverte, pp. 94-101.

Kergoat, Danièle (2009) Dynamique et consubstantialité des rapports sociaux, in Dorlin, E. (dir.) Sexe, race, classe, Paris, PUF, pp. 111-126.

Lada, Emmanuelle (2004) La fabrique de " l'ethnique » en entreprise publique, in Cossée, C. ; Lada, E. ; Rigoni, I. (dir.) Faire figure d'étranger, Paris, Armand Colin, pp. 139160.

Le Bihan, Yann (2007) Construction sociale et stigmatisation de la femme noire : imaginaires coloniaux et sélection matrimoniale, Paris, L'Harmattan, 428 p.

Le Bihan, Yann (2011) Femme noire en image, Paris, Éd. Hermann, 420 p.

Linhart, Danièle (2009) Travailler sans les autres ?, Paris, Seuil, 212 p.

Maruani, Margaret (dir.) (1998) Les Nouvelles frontières de l'inégalité. Hommes et femmes sur le marché du travail, Paris, La Découverte, 288 p.

Mathieu, Nicole-Claude (1971) Notes pour une définition sociologique des catégories de sexe, in Epistémologie sociologique, $\mathrm{n}^{\circ} 11$, pp. 1939.

Mathieu, Nicole-Claude (1984) De la conscience dominée des femmes, in Les Cabiers du GRIF, n²9, pp. 73-75.

Mathieu, Nicole-Claude (1985) Quand céder n'est pas consentir, in Mathieu, N.-C. (dir.) L'arraisonnement des femmes, Paris, Ed. de l'EHESS, pp. 169-245.

Mathieu, Nicole-Claude (1991) L'anatomie politique. Catégorisations et idéologies du sexe. Paris, Côté-Femmes, 293 p.

Mazrui, Alamin ; Mutunga, Willy (ed.) (2004) Governance and Leadership. Debating the African Condition, Ali Marrui and His Critics, Vol. 2, Londres, Africa Research \& Publications, 480 p.
Memmi, Albert (2002) Portrait du colonisé, précédé du portrait du colonisateur, Paris, Gallimard, 161 p.

Nkrumah, Kwame (2009) Le néo-colonialisme, dernier stade de l'impérialisme, Paris, Présence Africaine, $268 \mathrm{p}$.

Poiret, Christian (2005) Articuler les rapports de sexe, de classe et interethniques : quelques enseignements du débat nord américain, Revue Européenne des Migrations Internationales vol. 21, $\mathrm{n}^{\circ} 1$, pp. 195-226.

Poli, Alexandra (2004) Le renouvellement de l'action publique contre le racisme dans le cadre du dispositif 114/Codac, in Cossée, C. ; Lada, E. ; Rigoni, I. (dir.) Faire figure d'etranger, Paris, Armand Colin, pp. 211-225.

Perret, Thierry (2010) L'Afrique à l'écoute : la France, l'Afrique et la radio mondiale, Cabiers d'études africaines, n¹98-199-200, pp. 1003-1032.

Perret, Thierry (2005) Le temps des journalistes, Paris, Karthala, 318 p.

Preciado, Beatriz (2005)

Savoirs_Vampires@War, Multitudes,n²0 [en ligne, réf. du 28/06/2012] URL: $<\underline{\text { http://multitudes.samizdat.net/Savoirs- }}$ Vampires-War $>$.

Parameswaran, Radhika (2001) Feminist Media Ethnography in India : Exploring Power, Gender, and Culture in the Field, Qualitative Inquiry, Vol. 7, n¹, pp. 69-103.

Parameswaran, Radhika (2002a) Local Culture in Global Media : Excavating Colonial and Material Discourses in the National Geographic, Communication Theory, Vol. 12, n³, pp. 287-315.

Parameswaran, Radhika (2002b) Reading Fictions of Romance : Gender, sexuality, and Nationalism in Postcolonial India, Journal of communication, Vol. 52, n 4, pp. 832-851.

Parameswaran, Radhika (2008) The Other Sides of Globalization : Communication, Culture, and Postcolonial Critique, Communication, Culture \& Critique, $\mathrm{n}^{\circ} 1$, pp. 116-125.

Said, Edward (1980) L'orientalisme, Paris, Seuil, 422 p. 
Sanna, Maria-Eleonora (2006) Pratiques de soi et performance de genre : la construction des sujets politiques entre pouvoir et autonomie. Une lecture croisée de Michel Foucault et Judith Butler, Paris, Université Paris 8, 315 p. [En ligne, réf. du 28/06/2012] URL: <http://tel.archivesouvertes.fr/tel-00358609/fr/>.

Sartre, Jean-Paul (1956) Le colonialisme est un système, Les Temps Modernes, $\mathrm{n}^{\circ} 123$, pp.1371-1386 [en ligne, réf. du 06/12/2012] URL :

<http://socio13.wordpress.com/2009/03/3 $1 /$ le-colonialisme-est-un-systeme-parsartre/>.

Sosoe, Lukas K. (dir.) (2002) Diversité bumaine : Démocratie, multiculturalisme et citoyenneté, Paris, L'Harmattan, 569 p.

Spivak, Gayatri (2006) Les subalternes peuvent elles parler?, Paris, Ed. Amsterdam, 112 p.
Stearns, Peter N. (2008) Oxford Encyclopedia of the Modern World: 1750 to the Present [en ligne, réf. du 05/07/2012] URL: $<$ http://www.answers.com/topic/postcolo nialism $>$.

Verschave, François-Xavier (1999) La Françafrique, le plus long scandale de la République, Paris, Stock, 380 p.

Wasserman, Herman; $\mathrm{RaO}_{2}$ Shakuntala (2008) The Glocalization of Journalism Ethics, Journalism, Vol. 9, n²2, pp. 163-181. 